\title{
Ectopic Posterior Pituitary
}

National Cancer Institute

\section{Source}

National Cancer Institute. Ectopic Posterior Pituitary. NCI Thesaurus. Code C121151.

Atypically located posterior pituitary gland that may be associated with anterior or posterior pituitary hormone deficiencies. 